\title{
EXPERIMENTAL MUSEOLOGY: IMMERSIVE VISUALISATION AND CULTURAL (BIG) DATA
}

\section{Sarah Kenderdine}

In 1889, Smithsonian Institute curator George B. Goode (1891, p. 427) delivered an anticipatory lecture entitled 'The future of the museum' in which he forecast that the museum would one day 'stand side by side with the library and the laboratory.' As public cultural institutions, the primary mission of galleries, libraries, archives and museums is to provide citizens with knowledge not only about but through their collections and cultural heritage materials. With the advent of the Internet, from the mid-1990s the opportunities emerged for websites of public collections to become a virtual counterpart to the physical museum (Cameron \& Kenderdine, 2007; Kreiseler et al., 2017). Much has been made of the democratising potential of the digital transformation of museums (Museums and machines, 2016; Taylor \& Gibson, 2017). Paradoxically, the mass digitisation of public collections and their vast unseen annals, along with the concomitant metadata, has brought about an information overload that not only defies curation but also arguably further submerges the meaning of the archive in its own data (Vesna, 2007).

In recent decades, museum commentators have hinted that visualisation is a crucial intermediary between the digital archive and its big data, functioning both within galleries and beyond their physical location as networked access. A brief review of online cultural heritage collections reveals a visualisation revolution that requires rethinking the operational framework and the role of the museum in society (see, Windhager et al., 2019). At a deeper level, as Cui (2019) points out, information visualisation has itself altered how we view databases. Nonetheless, a large gap exists between what a human can do with data and what a machine might do. While this problem is often described in terms of a scalability challenge for visual analytics, in reality both human and machine limitations are at the root of this fundamental issue. Creating greater public engagement with collections through visualisation is not the magical solution for 
the problems facing museums - it is one step in a revolution of the way in which stories are told, and narrative unfolds.

While, on one level, visualisation is regarded as a simple means of communication, of a one-way information transfer, the critical frontier of advanced analytic tools, visualisations and situated interfaces are those that can bring audiences into meaningful communication with and creative co-production of cultural heritage. The museological turn toward a humanistic ethos has hardly been rapid. Interrogations began as far back as the 1980s, with the application of post-colonial critique to museums (see, Bennett, 1995, 2004), which occurred in parallel with the proposition that it might reinvent itself as the 'new' museum (Vergo, 1989). Since this time, museological and curatorial domains have been serially re-born as participatory, responsive, reflexive, inclusive, interrogative, relational and activist, with varying degrees of real structural change (see, Abungu, 2004; Butts, 2002; Chipangura \& Chipangura, 2020; Coleman, 2018; Mithlo, 2004; Vawda, 2019).

Experimental museology not only embraces this constantly changing landscape, it also challenges the mentality that feigns to 'open up' the museum through digitalisation while leaving intact its outdated, linear and canonical ethos as the chief custodian of heritage and authority on history. My own work in the field of experimental visualisation has made a departure from these institutional orthodoxies, as it has sought to transform public engagement with heritage through the application of aesthetic practice to cultural (big) data and the design of novel interactive frameworks. One of the earliest systems I created, The Virtual Room, was realised for Museum Victoria, Australia. Designed as a permanent gallery for situated experience in 2003, this stereographic interactive and immersive environment was one of the world's first large-scale visualisation systems for the mass public (see, Kenderdine \& Hart, 2003). I then went on to collaborate extensively with the iCinema Centre for Interactive Cinema Research at UNSW Australia and then to lead two research laboratories in the domain: the Applied Laboratory for Interactive Visualisation and Embodiment (ALiVE), Hong Kong, and UNSW Sydney's Expanded Perception and Interaction Centre (EPICentre).

In 2017, I established the Laboratory for Experimental Museology (eM+) at École polytechnique fédérale de Lausanne (EPFL) in Switzerland (Laboratory for Experimental Museology, n.d.). eM+ combines research from scientific, artistic and humanistic perspectives and promotes post-cinematic, multisensory experiences using experimental platforms. Its location in a 1,500-square-metre warehouse is home to nine large-scale visualisation systems, enabling transdisciplinary research at the intersection of aesthetics, immersive visualisation, interactive narrative and cultural data.

Despite such ground-breaking work, the expansion of the museological realm into the rich sensory, perceptual and social potential of experimental visualisation remains unchartered territory for many museums. This impasse was nowhere more evident after collecting organisations in 2020 around the world were closed to publics during the COVID-19 crisis, museum and gallery curators, directors and collection managers have been prompted to fling open the portals of their 
archives online. That a plethora of 'virtual visits' have plunged us into emptied gallery spaces illustrates just how many institutions have failed to fully understand the needs and desires of their audiences. In an era of networked digital culture, as set out by Hull and Scott (2013), many members of the public are able and ready to exploit the creative and participatory opportunities via the combined affordances of digital archives and social media.

Mapping out a possible path for such a future museum, this chapter elucidates some of the innovations in visualisation that I have developed in the domain of experimental museology, categorised as three approaches: collections visualisation, embodied visualisation and spatial and temporal visualisation. Before doing so, I provide here a brief overview of the state of the art of visualisation in the cultural heritage sector, the context out of which my own expertise continues to evolve.

\section{Visualisation and experimental museology}

Mapping data to visual representations has been used for centuries to reveal patterns, to communicate complex ideas and to tell stories. For Leonardo da Vinci, visuality in painting was the paragon of apprehension, surpassing both poetry and music. Daniel Albright (2014), in his theory of 'panaesthetics,' examines the way in which one art form can be translated to another (e.g. a painting is transformed into a musical composition).

Image-making for Harald Klinke (2014, p. 5) is not a 'simple process of externalisation of internal pictures - the process of drawing and painting [is] central to the process of thinking. It is not perception alone, but the complex process of picture-making that grasps reality and gives ideas about the world some sort of order.' At various junctures in the discourse of the humanities, scholars have pronounced new notions about how humans constitute reality. Both W.J.T. Mitchell's 'pictorial turn' (1994) and Erwin Panofsky's 'iconology' (1939) are theories that focussed on images rather than language. Ernst Cassirer, on the other hand, characterised images as 'giving sense to the world by symbolising ... experience in a process of perception and representation' (Cassirer quoted in Klinke, 2014 , p. 6). As such, and as Klinke contends (2014, p. 6), 'the question of images and their epistemic content ultimately points back to the human, who perceives, imagines and creates pictures. ... The power of images stems not from the images themselves, but from humans, who give them meaning.'

'Visualisation' encompasses these theories of the image and the function of their creation as a cognitive, transformative act. Visualisation, in the words of Scagnetti (2011), can be described as 'a medium for communication (or persuasion, or engagement)'; a tool for understanding (or problem solving, planning, orienting)'; a 'visual rhetoric' made of objects, including relations among those objects and tools for managing the relation between objects and environment; and as a 'visual epistemology' describing how we interpret the world. 'Information visualisation' is a graphical representation of (digital) data specifically designed to harness and augment basic powers of human perception for the task of comprehending large- 
scale information, and interactive visual representations of data are proven to further 'amplify cognition' (Card et al., 1999). Suffice to say, in response to digitisation, databases and networks, visualisation is becoming a dominant force through all disciplines. The application of digital visualisation techniques to cultural heritage data sets is today celebrated as a new and innovative research methodology (Bailey \& Pregill, 2014).

In defining visualisation as a representation, interpretive and revelatory, visualisation is both science and language. Like science, it represents data accurately and methodically, allowing us to detect underlying patterns, trends and relationships and, like language, it is used to convey meaning. Through visualisation, data is encoded into symbols and thus forms a system of semiotics. And yet visualisation poses specific problems for knowledge production.

As design and humanities scholar Johanna Drucker points out in the preface to her book Graphesis (2014), the reader of visualisations must learn the conventions of the diagrammatic knowledge form as this syntax is not inherent. These forms of visualisation may be infinitely varied and/or highly specific. In other words, graphic inscription itself is defined by characteristics that makes it hard to analyse. Unlike language, it is not a system that has a stable code, this makes visual analysis and visuality different from linguistics and language-based notational systems. Furthermore, images may conceal the decisions and processes on which they are based and appear to simply represent 'knowledge.' Visual representation reveals what is at stake in the distinction between information and interpretation within humanities practices. Drucker (2014, preface) argues that 'generative,' 'dynamic' and 'diagrammatic' images produce knowledge and that visualisations constitute information that possess the same legitimacy as any other human expression, such as written text. For Drucker, visualisations are 'graphical forms expressing interpretation' (2014, p. 54), and that because of the 'fundamentally interpreted condition on which data is constructed' (2014, p. 129) visualisations are a feature of both 'knowledge production and [its] presentation' (2014, p. 69).

The visualisation of cultural heritage collections began in the mid-1990s, as humanities research sought to expand the possibilities of descriptive and analytic data in object notation, metadata and the standard 'simple search' interface or inventory. On one hand, the constraints of relying on collection metadata as a search tool were immediately evident, it being uneven, unfinished and sometimes subjective. On the other hand, information retrieval itself, as underscored by Rogers et al. (2014), has presented serious limitations as a model for meaningful public engagement with cultural heritage collections.

Visualisation as an experimental approach is nonetheless unique in its potential to support all kinds of informal learning spaces. Cultural heritage collections comprise a potentially vast array of encounters within varied institutional settings, and experimental applications of visualisation have the potential to open up the museological realm to exploration both within and beyond the institutional walls. As outlined by Falk and Dierking (2019), the first methods to enhance the understanding of data through new visualisation literacies arose from research 
partnerships between science museums and educators, often with a specific focus on visual literacy or analytics as an enabler for scientific learning, study, or knowledge transfer (i.e., Kenderdine et al., 2016; Lock et al., 2018; Moss, 2019). Along these lines, visualisation has been developed as an effective tool for education, journalism and research knowledge transfer. Yet, when a visualisation is presented within the confines of the museum itself it is often curtailed in its capacity to respond to or collaborate with audiences, due to the fact that it is often employed to facilitate one-way communication, civic education or audience survey.

In contrast, creative approaches to interactive aesthetics and design have taken more inventive turns in the hands of artists (Jacobs et al., 2016). Countless examples of this rich field are documented in the proceedings of the Electronic Visualisation and Arts conferences (n.d.), which emerged from the Computer Arts Society, established in 1969, demonstrating the extent to which artists have been innovating throughout the entire modern history of computational science. Arguably evolving from media artists' initiatives, unique research partnerships have flourished in recent years, uniting creatives with educators, technologists, entertainment and gaming industries, heritage professionals and cultural institutions. These interdisciplinary alliances have fostered a burgeoning of innovation in sensory experiences via the creative exploration of multimodal technologies and dimensional realities, interface design, interactivity and data visualisation (Cantoni et al., 2019).

The domain of digital humanities emerged during the same decade (see, Schreibman et al., 2004; 2015). Collaboration between the digital humanities and electronic arts on the visualisation of cultural material and use of computational methods has however been very limited, a trend that Jänicke et al. (2017) identified in a study of the disciplinary crossover between the humanities and visualisation research communities between 2005 and 2015. Addressing this gap, Johanna Drucker $(2011 ; 2014 ; 2015)$ has comprehensively theorised the visual and visualisation in the digital humanities. Drucker (2014) specifically emphasises the role of visual cultures in contesting accepted forms of authority, through intellectual tools of imaginative thought, creative and aesthetic expression, as well as insisting on the role of subjectivity within all forms of human activity. Delineating the capacity of visualisation as a tool for critical response, Drucker (2013) points to a shift from the idea that the interface is an object, to its being the locus of interpretive activity, citing forensic features, distributed materiality, performative acts, enunciative dimensions and systemic ecologies as some of the potentially rich modalities of the medium.

In contrast, empiricist notions of data have, as Kitchin (2014) highlights, dominated mainstream information visualisation because they provide a convenient narrative for the aspirations of knowledge-oriented businesses in selling their services (e.g., for data brokers, data analytic providers, software vendors, consultancies). Along similar lines, Mayr and Windhager (2018, p. 3) delineate the drawbacks and benefits from a cognitive perspective of 'standard techniques for 
visually representing spatiotemporal data (coordinated linked views, animation or slideshows, layer superimposition, juxtaposition, and space-time cube representations).' As Chris Sula describes, visualisation can be used to aid decision making as well as facilitate collaboration, engaging new audiences and fostering higher levels of understanding. He extends a typical cognitive science approach to visualisation, amplifying the emotional, aesthetic, social, collaborative and shared responses to visualisations as fruitful areas of visualisation research for cultural heritage. In order to provide a counternarrative for information-based digital humanities, Drucker and Nowviskie (2004, para. 45) specifically adopt the term 'speculative,' to define approaches that 'make it possible for subjective interpretation to have a role in shaping the processes, not just the structures, of digital humanities.'

Visualisations have a crucial relationship to the physical realms of place, beings and matter - a dimension that has been sorely overlooked. Alongside the notion of digital materiality, an awareness is needed of the importance of the situated experience for knowledge transfer that engages both mind and body. Signalling the complexity of the domain of visualisation for museums, Windhager et al. (2019) argue that interactive visualisation must utilise both the screen and the onsite experience in a physical setting. Not et al. (2019) further this claim to promote tangible interaction as a means to augment digital artefacts, while Claes and Moere (2015) outline the role of tangible interaction on public information displays in increased information discovery.

Questions have however been raised by Kreiseler et al. (2017) regarding the quality of public engagement on offer in forms of visualisation designed with Web architecture in mind, and how explorable they actually make a collection. The increasing role of data science in this field has also brought with it ethical dilemmas, such as bias across the originating data and the algorithms and visualisations deployed to interpret and present it. These criticisms join calls for greater transparency, explainability and interpretability (Baur et al., 2020; Blackwell, 2015), a sentiment perhaps most aptly encapsulated in Whitelaw's (2015) proposition for more 'generous interfaces' for digital cultural collections. Ultimately, the need to overcome the data-centric view of automating knowledge and grapple with the human-computer interaction dimensions associated with a dynamic knowledge exchange remains intact (Wang et al., 2009).

The application of experimental museological approaches to visualisation engines is a significant new avenue for engagement with the mass data of cultural heritage. The experimental museology ethos provides an array of interactive models, tools and processes that support large-scale collections, including complexity, relationships, dynamism, aesthetics and embedded narratives (i.e., emergent interactive narrative). These approaches also harness various techniques aimed at engaging participants in sense-making, which can amplify cognition via affective modes and multi-modal interfaces that enable content-based adaptation to emotional experiences. These and other affordances are illustrated in the three approaches I am about to outline. 


\section{Collections visualisation}

Decades of digitisation have made a wealth of cultural material available online and offline. The Victoria and Albert Museum's collection offers over one million items, and Europeana's aggregated archives number some 31 million, while the National Library of Australia's Trove hosts around 128 million digitised newspaper articles. Many of the interfaces employed what Shneiderman (1996, p. 337) most famously heralded as the 'Visualisation Information-Seeking Mantra,' which entails 'overview first, zoom and filter, then details on demand.' More than 25 years later, this logic remains prevalent in the design strategies of websites of cultural organisations.

The ways that collections can be reassembled, mined and experienced are proliferating as are paradigm-changing technologies such as machine learning, computer vision and novel visualisations. Digital tools for the past decade have in turn enabled a wide array of interaction technique. On the one hand, visualisation of cultural heritage data has to encompass sense-making, from foraging to synthesis. On the other hand, the integration of algorithms and visualisation techniques for large amounts of data in visual analytics can be applied as part of these interactive aspects to help reduce the cognitive burden of searching as well as the mismatch between data size and complexity, and human acuity (Cui, 2019).

I was presented in 2011 with the challenge of creating a database for approximately 100,000 objects, a small subset of the 16 million records held in Museum Victoria's vast collections (mArchive). As part of this endeavour, I established a number of new concepts for visualisation, including the notion of 'cultural data sculpting' (Kenderdine et al., 2012), where users are provided with multimodal analytical tools to shape heterogeneous datasets through their visualisation in an interactive and immersive environment (Kenderdine et al., 2012). Sculpting information in this way specifically enables users to 'explore cultural data as a cultural artifact so as to expose a multiplicity of narratives that may be arranged and projected instantaneously atop the data archive architecture and its metadata' (Kenderdine et al., 2012, p. 205). The resulting application took the form of a real-time curating machine and a shared playground for interaction that opened up the museum storehouse. The non-text-based interactive collections engine was installed in an omnidirectional, omnispatial virtual environment designed for about 30 people at any one time (Kenderdine \& Hart, 2011; 2014). T_Visionarium, is yet another example that reveals the power of aesthetic transcription as a fundamental parameter for archival reuse. Developed over various iterations from 2004 to 2009 at UNSW Sydney's iCinema, the core of the project was 24 hours of television footage. Following machine and human analysis and classification of this footage, a database of 24,000 hours of segments was established. As the viewer interacted, the 3D panoramic display of clips would automatically generate a live re-composition of the archive in a transnarrative experience (see Bennett, 2007).

Exposing the structure and processes of subjective interpretation through visual means are at the heart of cultural visualisation. Yet, as visual representations are 
used with greater frequency within the digital humanities, the instability of graphical systems are too often bracketed in a rush to make use of visual conventions with roots in the representation of statistical information. As a result, the interpretative richness of visuality gets sacrificed as well. And these forms of visualisation are certainly not suited to the fundamental process of doing interpretation in visual form. What cultural collections need most of all is to apply a humanistic model of interface and interaction that emphasises exploration and interpretation over task and information retrieval.

Overcoming these barriers to engagement was a primary interest in the works exhibition, Infinity Room 2, presented in 2019 at ArtLab EPFL, Lausanne. Celebrating the 50th anniversary of EPFL's foundation, the exhibition transformed some of the school's vast archives, records and assemblages into a series of experimental visualisations. The first of the total of eight installations, excavated the collection Open Science to create an eclectic assemblage of fifty iconic objects from EPFL, exhibited as an array of 'augmented' storage lockers. Using a tablet as an augmented reality interface, participants were able to 'open' a series of doors, each revealing an unexpected 3D object within, as a contemporary Wunderkammer of art and science.

Another major EPFL collection, the Alain Herzog Archive is a sweeping photographic vision of campus life via half a million images taken over a quarter century. With no extant metadata available except for dates, the collection was visualised for the installation using machine learning algorithms to hone it into themes of science or architecture, which were then narrowed into a range of subthemes, including robots, portraits and more. Oscillating between 'distant reading' patterns deduced from the coherence of the tagging, through to largescale projections of single images, this process recomposited the archive, moving between recognisable classifications and surprising juxtapositions, sometimes with humorous results for EPFL staff, students and alumni.

The centrepiece of Infinity Room 2, Jazz Luminaries situated viewers within a social network constellation of the jazz greats in the Montreux Jazz Archive. The work transforms this UNESCO Memory of the World collection, recently digitised at EPFL, into an interactive installation, which combines machine learning, computer vision and novel visualisations, all of which are engaging artists, scientists and the public who are able to generate new meanings out of the archive. Reclined under the full dome in a hemispheric gestalt, participants in Jazz Luminaries were able to generate an experience of their own, to unfold the social network of more than 5,400 musicians whose recordings are held in the Montreux Jazz Archive (Montreux, n.d.).

Through a unique multimodal interface Jazz Luminaries allows viewers to cut, remix and replay over 13,000 videos of those 5,400 jazz greats, displayed in a full dome measuring six metres in diameter. Each musician is represented by a node, which is interconnected to other Montreux Jazz Festival artists based on their historic collaboration over the years. Navigating this vast archive enlivens the constellation of relationships between these artists. The proximity of the nodes in 
the network (and thus their link strength) are based on the number of times any one musician played with another artist at the festival. At the centre of this universe is the legendary B.B. King, the 'King of the Blues,' who jammed with countless others having, as The Sydney Morning Herald reported in 2006, appeared at Montreux for more than 20 years (Figures 1.1 and 1.2).

Hermetically sealed in personalised headphones, twelve participants at a time lounge beneath the jazz 'star' map under a spherical dome. A spherical ball acts as the interface and its operations replace the text-based search with auditory strategies of 'tuning.' This interface emulates the curvature of the dome itself, which has an ability to completely envelope the 'spherical gestalt of the human visual field,' as McConville claimed (2007, p. 77). Similar to radio channel surfing, participants move the selector over the nodes, and the move serves to activate the sound files linked to each artist as well as those of their collaborators. Surfing produces a rapidly changing sonic cloud or an anarchic assemblage of clips. Cutting between discovered video fragments, the participant can select those with the most intensity or reciprocity. Choosing the clip again rewards them with the performance in its entirety. Shedding its original framed recorded form, the footage explodes into a fractal of perspectives.

The participant performs this 'remix' for all the other people who recline around them under the dome. As they surf, they are intimately aware that they are sharing a selection with their counterparts. Socialising the interface in this way, those gathered lay back and enjoy a vibrant unfolding of the Montreux Jazz Archive at the hands, and ears, of others. Centred on properties of recollection, regeneration and reworking, as well as rich modes of visualisation, interaction engagement and serendipitous discovery, Jazz Luminaries marks the shift from a linear classification of objects within inventories to their remix. It embodies the paradigmatic move from the traditional model of stewardship (of curation and managed access) to one of co-production and new forms of distributed authority.

This future has yet to arrive in the museological mainstream. Windhager et al. (2016, p. 75) underscore that 'digital interfaces mostly strive to augment and enrich traditional in situ-interaction with collections,' while the remote exploration of cultural collections on screens still falls short of the in-situ experience of a museum collection. The full potential of visualisation requires designing specific suitable prosthetic architectures that place audiences at the centre of the archive.

\section{Embodied visualisation}

The communication and transmission of cultural expression crucially relies on enacted practices, which are intimately linked through people and their physical being. Perpetuated through forms including performance, dance, song or ritual acts, such heritage is kept alive through repeated, ever-changing acts, which form 'repertoires' (Taylor, 2003). In some instances, however, such practices have vanished, due to the rupture of a community's cultural transmission. In other 


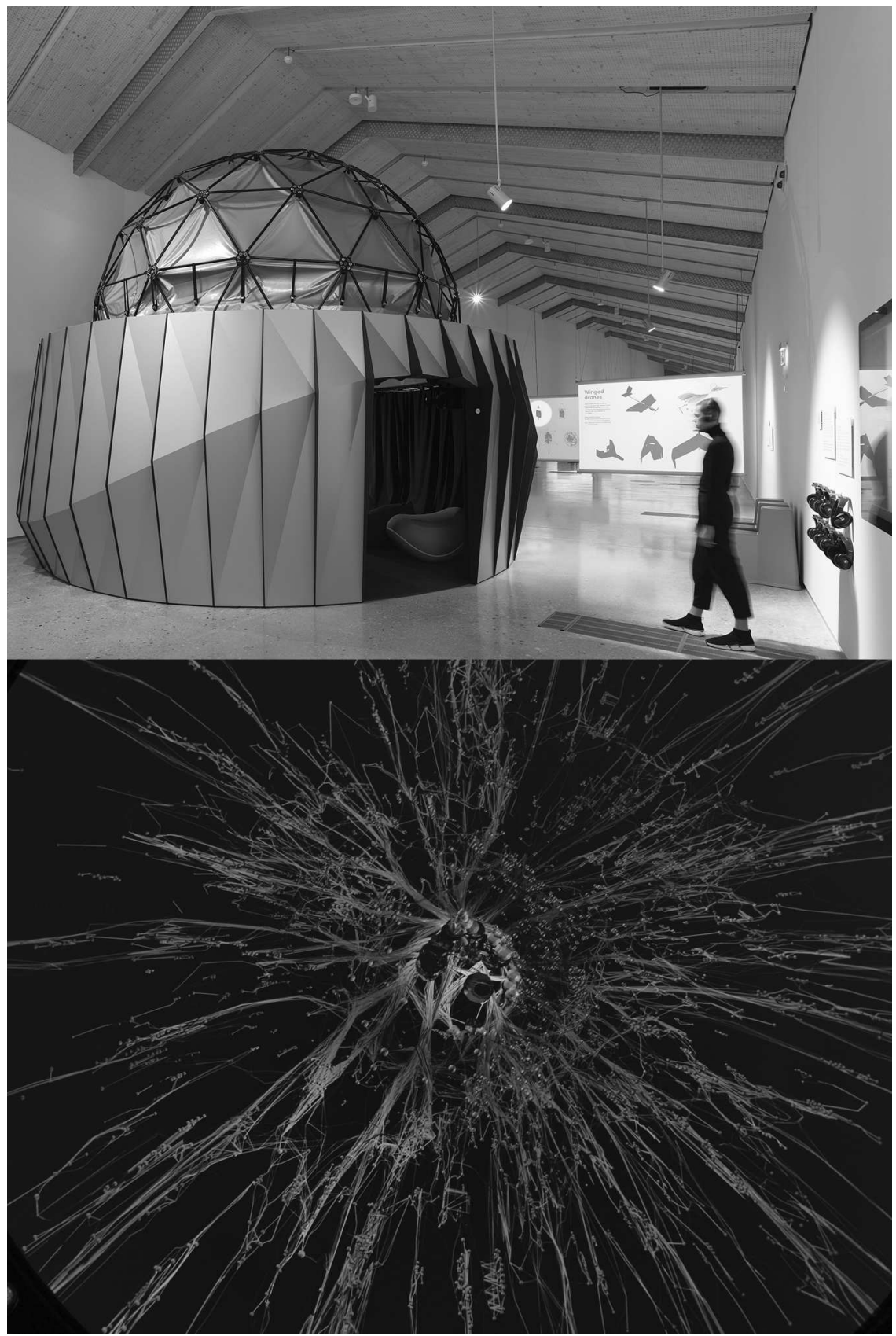

FIGURE 1.1 Jazz Luminaries, Infinity Room II, ArtLab 2019. Photo: Catherine Leutenegger. 


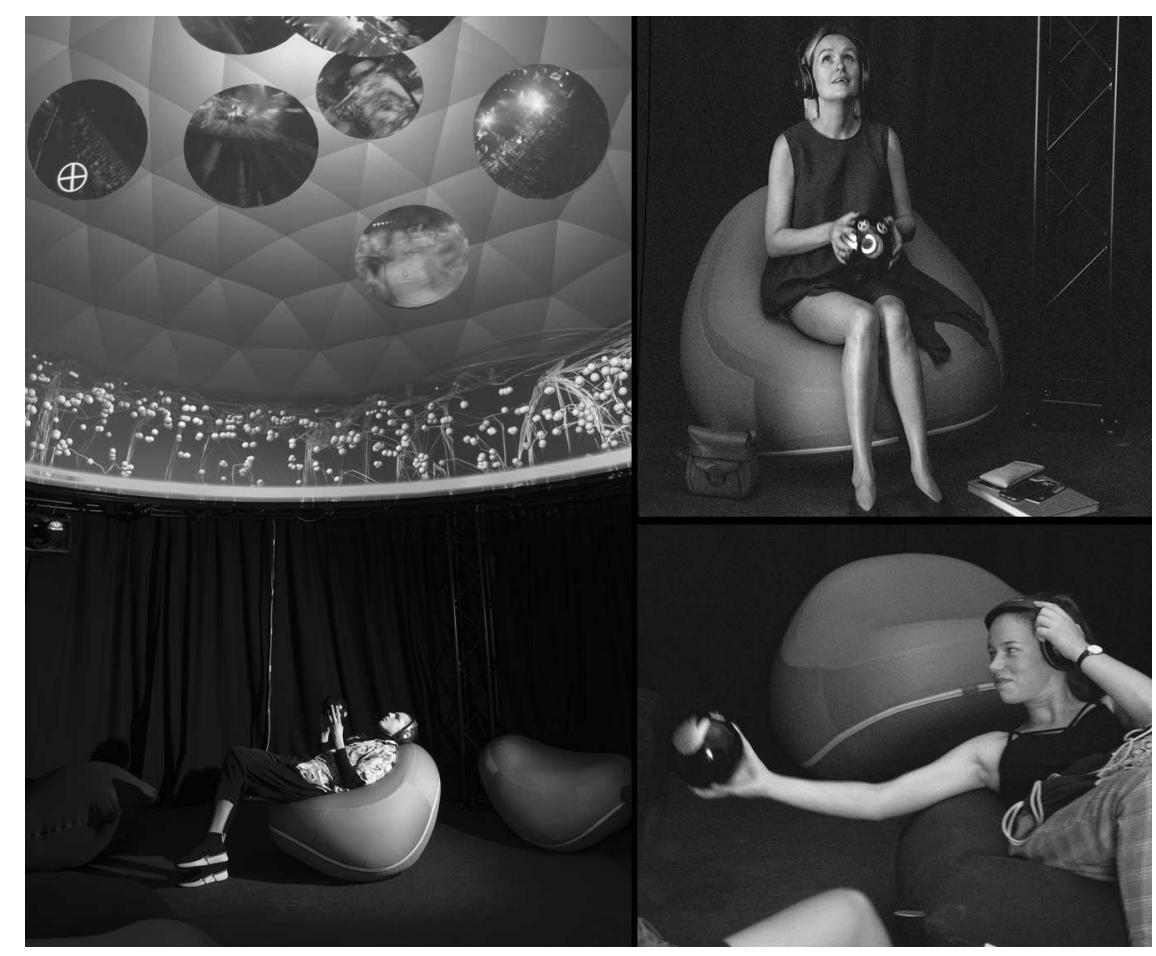

FIGURE 1.2 Jazz Luminaries, Infinity Room II, ArtLab 2019. Photos: Catherine Leutenegger/Sarah Kenderdine.

instances, there has been an appropriation or revival of a practice from the past, as in the case of popular historical re-enactment, but even these forms feature attributes of tacit and embodied expert knowledge.

As the domain of intangible heritage has garnered increasing scholarly attention, the theorisation of the body as a repository of knowledge has begun to consolidate across a number of disciplines. Although the notion is not new in performance studies, a novel understanding has recently emerged that view performers' or actors' embodied capacity as generative archives. The premise is that otherwise inaccessible knowledge can be unlocked via embodied acts - also called 'embodied historiography' (Branch \& Hughes, 2014; Johnson, 2015). This notion segues into my own work concerning embodied knowledge archives and their experimental visualisation (Chao et al., 2018; Kenderdine, 2015; Kenderdine \& Hart, 2014). The primary visualisation methodologies I have developed as part of a novel embodied historiography centre on the means to create a digital record of tacit knowledge. Most of all, this documentation must be accessible to others at a later date, especially without the presence of the original expert to demonstrate or teach that knowledge.

A prime example of this work is the Hong Kong Martial Arts Living Archive (HKMALA), established in 2012, which applies new digital methods to 
reconstruct, analyse and transmit Chinese embodied heritage. HKMALA is an ongoing research collaboration between the International Guoshu Association, City University of Hong Kong, and eM+ at EPFL. From the early- to mid-20th century, Hong Kong provided refuge to teeming thousands of immigrants from mainland China, and among them were some of the most prominent martial artists in the world. With globalisation, urbanisation and a dwindling number of practitioners, this living heritage is in danger of being lost. Kung fu hinges on personto-person exchange between an expert and a novice and requires the imitation of movements of a master or an instructor (Chan et al., 2011; Komura et al., 2006). For this reason, HKMALA undertakes its unique approach to embodied historiography through performance-based reconstruction to build an archive that currently contains nineteen styles by 33 elite practitioners and over 130 motion capture datasets. As part of the endeavour to institute the body as the principal site for embodied knowledge, we have developed a range of digital prostheses, from using life-size models and 3D interfaces, to interactive real-time applications in large-scale virtual environments (Chao et al., 2016; 2018; Kenderdine \& Shaw, 2016; 2018).

Multimodal participation is a crucial tool, manifest in the HKMALA Pose Matching installation. Featured in the ArtLab exhibition Kung Fu Motion in 2018, on a human-scaled projection screen, the participant is prompted to take up the stance of a 'master.' Once in position, sensors 'motion capture' their movement and body position. These are matched, with a video sequence of poses presented on the screen, originally performed by a kung fu master. As the participant configures their body to match the poses, a corporeal conjunction is created, in which the somatic memory of the kung fu master is imprinted on their body. Beyond its playful aspect, this installation could be an invaluable teaching and learning tool for current and future generations of kung fu practitioners (Lindgren \& Johnson-Glenberg, 2013).

A number of the HKMALA datasets have been reconfigured as interactive environments, supporting research showing that immersive visualisations offer dynamic situations for learning and embodied cognition (Stefaniak, 2014). Situated in the Re-ACTOR system, a six-sided, panoptic virtual reality environment at $\mathrm{eM}+$, the Kung $\mathrm{Fu}$ Visualisation brings together historical materials with creative visualisations of one of the kung fu master's reenacted performances. On each of Re-ACTOR's six sides, an interactive control panel allows visitors to view six different visualisation styles, elucidating the underlying dynamics of the master's movements. These visualisations deploy advanced documentation processes, including motion capture, motion-over-time analytics, 3D reconstruction and panoramic video, exposing the depth and array of intricate dynamics in the embodied repertoire (Figure 1.3).

This project also harnesses the fast-paced developments arising in cinema and game industries, such as volumetric video and $3 \mathrm{D}$ animation based on motion capture. The Digital reconstruction of Lam Sai Wing (2018), is a powerful example of what is possible in the digital reconstruction of archives of embodied 


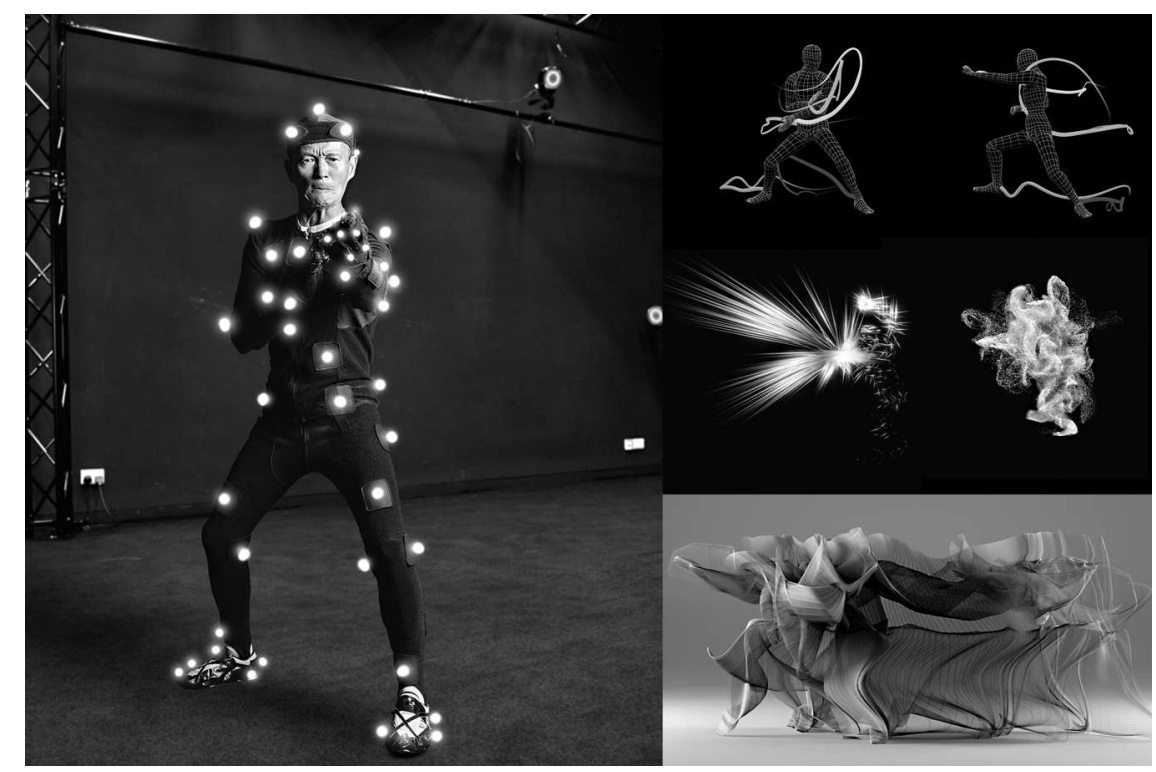

FIGURE 1.3 Hong Kong Martial Arts Living Archive: motion capture and Kung Fu Visualisation. Photos: Sarah Kenderdine.

transmission. Lam Sai Wing was one of the most important early-20th century kung fu master practitioners of the south Chinese tradition in Hong Kong. Taking up technologies that have perfected the manufacture of 3D human avatars, the virtual reconstruction of the late master's facial and bodily features from old photographic portraits were mapped onto a sequence of his martial arts movements. While, in yet another example of the power of visualisation for transmission, these moves were simulated with data extracted from contemporary reenactments performed by his descendant, Master Oscar Lam.

The ongoing difficulty of ensuring such heritage can be re-performed and that its repertoire is made available to the body is a major challenge for experimental museology. It is, however, promising that the visualisation of embodied acts provides the opportunity for both the documentation and reproduction of tacit knowledge for future generations.

\section{Spatial and temporal visualisation}

The final part of this chapter presents some of my work on post-cartographic and deep mapping, which has sought to reconstruct the role of narrative in the visualisation of vast temporally and spatially distributed sites and objects. Early visualisation projects in this domain feature a 3D, 360-degree panoramic screen, augmented in order to enliven participants within an interactive 'omnidirectional' environment. PLACE-Hampi, for example, is viewed from a motorised platform, which a single visitor is able to control while up to 25 people are in the space in 
360 degrees and 3D (see, Kenderdine, 2013). Focussed on significant archaeological, historical and sacred locations of the World Heritage Site Vijayanagara (Hampi), South India, PLACE-Hampi creates an embodied theatre of participation in the drama of Hindu mythology (see, Kenderdine, 2007). Comprising highresolution augmented stereoscopic panoramas and surrounded by a rich sound field of ambisonic recordings, the viewer in PLACE-Hampi is co-present in their narrative rediscovery of the cultural landscape.

As critical cartographers Kitchin and Dodge (2007) have argued, maps are always in the state of becoming, brought into being through embodied social and technical practices to solve relational problems such as plotting, planning or navigating. Maps of this kind thus emerge through a mix of creative, reflexive, playful, tactile and habitual practices as a co-constitutive process - a production that is constantly in motion. In parallel, according to Buchmüller et al. (2019), visualisation design is moving from presenting big data as a mosaic, a river or series of ribbons, in a map, fixed in time, onto a moving stream or rug that captures collective movement and spatial-temporal dimensions dynamically.

An example of this approach is the Atlas of Maritime Buddhism, a large-scale mapping project that I began in 2016. The aim of the Atlas is to relate the story of the spread of Buddhism from India through the seaports of Southeast Asia and South China Sea, which triggered a profusion of cross-cultural exchanges that had a profound impact on Asian and world history.

The Atlas comprises multiple overlapping chronological events, supported by archaeological and historical evidence that has never been brought together before from disparate spatial locations represented by approximately 170 generalised information layers. With contributions from researchers around the world, it includes geospatial coordinates, gazetteers for hundreds of sites; images of archaeological sites and artefacts, religious and geopolitical empires and zones of influence, inscriptions and transcriptions of Sanskrit texts, historic maps, accounts by Buddhist monks and ambassadors, records of trade, hydrographic data, monsoon records and shipwreck datasets. Thousands of locations have also been recorded in ultra-high resolution 3D panoramic and spherical imaging across hundreds of world heritage sites, spread across 12 countries. In addition, hundreds of priceless sculptures from national and local museums have been modelled in 3D, to create an extraordinary survey of iconographic transformation throughout the region.

Given this vast heterogeneity, the Atlas has demanded a new form of visual, cartographic and time-space narrative strategy, beyond traditional forms of interpretation (see, Presner \& Shepard, 2015). The schema for Atlas supports narrative construction via the world's first deep-mapping data browser - an interface for exploring narrative patterns, processes and phenomena. This deep mapping schema converts the chart of information from a mimetic object (based on a perceived territory) into a navigational one, where 'everything is on the move' (November et al., 2010, p. 595). Conceiving of maps through a deep-mapping schema, following Ridge et al. (2013), assumes that they are never fully formed (or 
static) but that they instead emerge in dynamic navigational processes where emergent narrative formations are possible.

The Atlas then comes to life in its specially conceived 360-degree, omnidirectional panoramic environment, as an interpretive tool for the space in which culture and body converge within a topological map of place. In this way, the visualisation of cultural cartography in the Atlas of Maritime Buddhism opens onto a thousand plateaus where the stories can be remapped in myriad dimensions and directions, demonstrating the potential for visualisation to enact a powerful rethinking of museological objects as deep maps of space and time. Such a reimagining of maps fundamentally changes the focus of cartography, away from notions of accuracy, design, aesthetics and power. It shifts mapping toward the complex, contingent interactions between the researchers collecting the data, the designers of the information visualisation system, its users, geo-temporal data and the nonphysical aspects of place. The reconfiguration of this vast data set as an immersive, interactive 'deep map' addresses the fundamental challenges of narrative coherence for museum audiences exploring digital cultural atlases.

\section{Conclusion}

In this chapter, I have provided a panorama of the changing landscape of visualisation as it is emerging from my own work in experimental museology, in conjunction with recent research in data science and the digital humanities. I have also demonstrated that the influence and input from artists has also been important to the progress in visualisation. What is evident today is that many cultural institutions have taken great pains to become more participatory and audience focussed, in tandem with a shift of principles within heritage science. The framework of experimental museology offers ideas, creativity and tools needed for the application of visualisation to cultural heritage collections. However, more interdisciplinary work remains to be done to ascertain how and where physicaldigital interaction in big data visualisation and technology design can be balanced and will effectively support public engagement and learning, experience and ability. To reconfigure the future museum as an avenue for sensory discovery, openness and participation, that incorporates embodied as well deep mapping approaches, new avenues must be continuously generated.

\section{Bibliography}

Abungu, G. (2004). Democratising museums and heritage: Ten years on. South African Museums Association Bulletin, 30(1), 3-5.

Albright, D. (2014). Panaesthetics: On the unity and diversity of the arts. Yale University Press.

Bailey, J., \& Pregill, L. (2014). Speak to the eyes: The history and practice of information visualization. Art Documentation: Journal of the Art Libraries Society of North America, 33(2), 168-191. 
Baur, T., Heimerl, A., Lingenfelser, F., Wagner, J., Valstar, M. F., Schuller, B., \& André, E. (2020). eXplainable cooperative machine learning with NOVA. KI - Künstliche Intelligenz, 34, 143-164.

Bennett, T. (1995). The birth of the museum: History, theory, politics. Routledge.

Bennett, T. (2004). Pasts beyond memories: Evolution, museums, colonialism. Routledge.

Bennett, J. (2007). T_Visionarium: A user's guide. UNSW Press/ZKM.

Blackwell, A. F. (2015). Interacting with an inferred world: The challenge of machine learning for humane computer interaction. Aarhus Series on Human Centered Computing, 1(1), 1-12.

Bradley, K. (2015). Why museums hide masterpieces away. BBC Culture online. 23 January 2015. http://www.bbc.com/culture/story/20150123-7-masterpieces-you-cant-see

Branch, B., \& Hughes, E. (2014). Embodied historiography: Rupture as the performance of history. Performance Research, 19(6), 108-115.

Buchmüller, J., Jäckle, D., Cakmak, E., Brandes, U., \& Keim, D. A. (2019). MotionRugs: Visualizing collective trends in space and time. IEEE Transactions on Visualization and Computer Graphics.

Butts, D. (2002). Māori and museums: The politics of indigenous recognition. In R. Sandell, (Ed.), Museums, society, inequality (pp. 225-243). Routledge.

Cameron, F., \& Kenderdine, S. (2007). Theorizing digital cultural heritage: A critical discourse. MIT Press.

Cantoni, V., Mosconi, M., \& Setti, A. (2019). Technological innovation and its enhancement of cultural heritage. 2019 IEEE International Symposium on Innovations in Intelligent SysTems and Applications (INISTA), 1-6.

Card, S.K., Mackinlay, J., \& Shneiderman, B. (1999). Readings in information visualization: Using vision to think. Morgan Kaufmann.

Cassirer, E. (1923-1925). The philosophy of symbolic forms (J. M. Krois, Trans.), 4 volumes. Yale University Press.

Chipangura, N., \& Chipangura, P. (2020). Community museums and rethinking the colonial frame of national museums in Zimbabwe. Museum Management and Curatorship, 35(1), 36-56.

Claes, S., \& Moere, A. V. (2015). The role of tangible interaction in exploring information on public visualization displays. Proceedings of the 4th International Symposium on Pervasive Displays, 201-207.

Chan, J., Leung, H., Tang, J., \& Komura, T. (2011). A virtual reality dance training system using motion capture technology. IEEE Transactions on Learning Technologies, 4(2), 187-195.

Chao,H., Kenderdine, S., \& Shaw, J. (Eds). (2016). 300 Years of Hakka Kung Fu: Digital vision of its legacy and future. International Guoshu Association.

Chao, H., Delbridge, M., Kenderdine, S., Nicholson, L., \& Shaw, J. (2018). Kapturing Kung Fu: Future proofing the Hong Kong Martial Arts Living Archive. In S. Whatley, R. Cisneros, \& A. Sabiescu (Eds.), Digital echoes (pp. 249-264). Palgrave.

Coleman, L. (2018). Understanding and implementing inclusion in museums. Rowman \& Littlefield.

Cui, W. (2019). Visual analytics: A comprehensive overview. IEEE Access, 7, 81555-81573. Scopus.

Davey, E. (2010, 31 March). The 99\% of the British Museum not on show. BBC London online. http://news.bbc.co.uk/local/london/hi/people_and_places/arts_and_culture/ newsid_8595000/8595594.stm 
Digital reconstruction of Lam Sai Wing, (2018). Created for the Hong Kong Martial Arts Living Archive (HKMALA), by Sarah Kenderdine's Laboratory for Experimental Museology $(\mathrm{eM}+)$ at EPFL and Jeffrey Shaw of the CityU Hong Kong.

Drucker, J. (2011). Humanities approaches to graphical display. Digital Humanities Quarterly $5(1), 1-21$.

Drucker, J. (2013). Performative materiality and theoretical approaches to interface. DHQ: Digital Humanities Quarterly, 7(1), 1.

Drucker, J. (2014). Graphesis: Visual forms of knowledge production. Harvard University Press.

Drucker, J. (2015). Graphical approaches to the digital humanities. In S. Schreibman, R. Siemens, \& J. Unsworth (Eds.), A new companion to digital humanities (pp. 238-250). John Wiley \& Sons.

Drucker, J., \& Nowviskie, B. (2004). Speculative computing: Aesthetic provocations in humanities computing. In S. Schreibman, R. Siemens, \& J. Unsworth (Eds.), A companion to digital humanities. Blackwell.

Falk, J. H. \& Dierking, L. D. (2019). Reimagining public science education: the role of lifelong free-choice learning. Disciplinary and Interdisciplinary Science Education Research, 1(10). https:// doi.org/10.1186/s43031-019-0013-x

Goode, G. B. (1891). The museums of the future. Smithsonian Institution.

Hull, G., \& Scott, J. (2013). Curating and creating online: Identity, authorship, and viewing in a digital age. In K. Drotner \& K. C. Schrøder (Eds.), Museum communication and social media (pp. 130-150). Routledge.

Jacobs, R., Benford, S., Luger, E., \& Howarth, C. (2016). The prediction machine: Performing scientific and artistic process. Proceedings of the 2016 ACM Conference on Designing Interactive Systems, 497-508.

Jänicke, S., Franzini, G., Cheema, M. F., \& Scheuermann, G. (2017). Visual text analysis in digital humanities. Computer Graphics Forum, 36(6), 226-250.

Johnson, K. (2015). Rethinking (re)doing: Historical re-enactment and/as historiography. Rethinking History, 19(2), 193-206.

Kenderdine, S. (2007). Somatic solidarity, magical realism and animating popular gods: Place-Hampi 'where intensities are felt.' Proceedings, 11th International Conference on Information Visualisation, N.4272012, 402-408.

Kenderdine, S. (2008). An entanglement of people-things: Place-Hampi. International Journal of Digital Cultural Heritage and E-Tourism, 1(2-3), 139-156.

Kenderdine, S. (2013). Place-Hampi: Inhabiting the panoramic imaginary of Vijayanagara. Kehrer Verlag.

Kenderdine, S. (2015) Embodiment, entanglement and immersion in digital cultural heritage. In S. Schreibman, R. Siemens, \& J. Unsworth (Eds.), A new companion to digital humanities (pp. 22-41). Wiley-Blackwell.

Kenderdine, S., \& Hart, T. (2003). This is not a peep show! The virtual room at the Melbourne Museum. Proceedings of International Committee on Hypermedia and Interactivity, Paris September, Pittsburgh: Museum Archives and Informatics. CDROM.

Kenderdine, S., \& Hart, T. (2011). Cultural data sculpting: Omni-spatial visualization for large scale heterogeneous datasets. Museums and the Web 2011: Proceedings, Philadelphia, 6-9 April. https://www.museumsandtheweb.com/mw2011/papers/cultural_data_sculpting omni_spatial_visualiza.html

Kenderdine, S., \& Hart, T. (2014). mARChive: Sculpting Museum Victoria's collections. Museums and the Web: Proceedings, 2-5 April, Baltimore, USA. https://mw2014. museumsandtheweb.com/paper/marchive-sculpting-museum-victorias-collections/ 
Kenderdine, S., Nicholson, J., \& Mason, I. (2016). Modelling people and populations: Exploring medical visualization through immersive interactive virtual environments. In J. Nicholson, A. Darzi, E. Holmes, \& J. C. Lindon (Eds.), Metabolic phenotyping in personalized and public healthcare (pp. 333-367). Elsevier.

Kenderdine, S., \& Shaw, J. (2016). A digital legacy for Living Culture. In H. Chao, S. Kenderdine, \& J. Shaw (Eds.), 300 Years of Hakka Kung Fu: Digital vision of its legacy and future. International Guoshu Association.

Kenderdine, S., \& Shaw, J. (2018). The museological re-enactment of Lingnan Hung Kuen. In H. Chao (Ed.), Lingnan Hung Kuen across the century: Kung Fu narratives in cinema and community (pp. 137-159). City University of Hong Kong Press.

Kenderdine, S., Shaw, J., \& Gremmler, T. (2012). Cultural data sculpting: Omnidirectional visualization for cultural datasets. In F. Marchese \& E. Banissi (Eds.), Knowledge visualization currents: From text to art to culture (pp. 199-221). Springer.

Kitchin, R. (2014). Big data, new epistemologies and paradigm shifts. Big Data \& Society, $1(1), 1-12$.

Kitchin, R., \& Dodge, M. (2007). Rethinking maps. Progress in Human Geography, 31(3), 331-344.

Klinke, H. (2014). Introduction: The image and the mind. In H. Klinke (Ed.), Art theory as visual epistemology (pp. 1-10). Cambridge Scholars Publishing.

Komura, T., Lam, B., Lau, R. W., \& Leung, H. (2006). E-Learning martial arts. International Conference on Web-Based Learning (pp. 239-248). Springer.

Kreiseler, S., Brüggemann, V., \& Dörk, M. (2017). Tracing exploratory modes in digital collections of museum Web sites using reverse information architecture. First Monday, 22(4-3). https://firstmonday.org/ojs/index.php/fm/article/download/6984/6090

Laboratory for Experimental Museology. (n.d.). https://www.epfl.ch/labs/emplus/

Lindgren, R., \& Johnson-Glenberg, M. (2013). Emboldened by embodiment: Six precepts for research on embodied learning and mixed reality. Educational Researcher, 42(8), $445-452$.

Lock, J. G., Filonik, D., Lawther, R., Pather, N., Gaus, K., Kenderdine, S., \& Bednarz, T. (2018). Visual analytics of single cell microscopy data using a collaborative immersive environment. Proceedings 16th SIGGRAPH International Conference, Article no. 1.

Mayr, E., \& Windhager, F. (2018). Once upon a spacetime: Visual storytelling in cognitive and geotemporal information spaces. ISPRS International Journal of Geo-Information, 7(3), 96.

McConville, D. (2007). Cosmological cinema: Pedagogy, propaganda, and perturbation in early dome theaters. Technoetic Arts, 5(82), 69-85.

Mitchell, W. J. T. (1994). Picture theory: Essays on verbal and visual representation. University of Chicago Press.

Mithlo, N. M. (2004). 'Red man's burden': The politics of inclusion in museum settings. American Indian Quarterly, 28(3-4), 743-763.

Montreux Jazz Digital Project. (n.d.). https://www.epfl.ch/innovation/domains/culturalheritage-and-innovation-center/montreux-jazz-digital-project-2/

Moss, K. E. (2019). A beacon for broader impact: Illuminating science. Journal of Visual Communication in Medicine, 42(2), 66-75.

Museums and machines: Curating tech to democratize art, culture, history and science. (2016, October 17). Transform. https://news.microsoft.com/transform/museums-andmachines-curating-tech-to-democratize-art-culture-history-and-science/

Not, E., Cavada, D., Maule, S., Pisetti, A., \& Venturini, A. (2019). Digital augmentation of historical objects through tangible interaction. Journal on Computing and Cultural Heritage, 12(3), 18:1-18:19. 
November, V., Camacho-Hübner, E., \& Latour, B. (2010). Entering a risky territory: Space in the age of digital navigation. Society and Space, 28(4), 581-599.

Panofsky, E. (1939). Studies in iconology: Humanistic themes in the art of the renaissance (facsimile repr., 1927). Oxford University Press.

Presner, T., \& Shepard, D. (2015). Mapping the geospatial turn in A New Companion to Digital Humanities. In S. Schreibman, R. Siemens, \& J. Unsworth (Eds.), A companion to digital humanities (pp. 199-212). Blackwell.

Ridge, M., Lafreniere, D., \& Nesbit, S. (2013). Creating deep maps and spatial narratives through design. International Journal of Humanities and Arts Computing, 7(1-2), 176-189.

Rogers, K., Hinrichs, U., \& Quigley, A. (2014). It doesn't compare to being there: In-situ vs. remote exploration of museum collections. https://research-repository.st-andrews.ac.uk/ handle/10023/7239

Scagnetti, G. (2011). Visual epistemology for design in complex systems, lecture at Tecnológica de Monterrey: Academic leaders' program. http://namedgaia.com/index. php?/teaching/visual-epistemology/

Schreibman, S., Siemens, R., \& Unsworth, J. (Eds.), (2004). The digital humanities and humanities computing: An introduction, A companion to digital humanities (pp. xxiii-xxvii). Blackwell.

Schreibman, S., Siemens, R., \& Unsworth, J. (Eds.). (2015). A new companion to digital humanities. Wiley-Blackwell.

Shneiderman, B. (1996). The eyes have it: A task by data type taxonomy for information visualizations. Proc. IEEE. Visual Lang. Art. no. 336.

Simon, N. (2010). The participatory museum. Museum 2.0.

Stefaniak, J. (2014). Immersive simulation: The replication of environments to practice problem solving. In M. Gosper \& D. Ifenthaler (Eds.), Curriculum models for the 21st century: Using learning technologies in higher education (pp. 313-324). Springer.

Sula, C. A. (2013). Quantifying culture: The value of visualization inside (and outside) libraries, museums, and the academy. In J. P. Bowen, S. Keene, \& K. Ng (Eds.), Electronic visualisation in arts and culture (pp. 25-37). Springer.

Taylor, D. (2003). The archive and the repertoire: Performing cultural memory in the Americas. Duke University Press.

Taylor, J., \& Gibson, L. K. (2017). Digitisation, digital interaction and social media: Embedded barriers to democratic heritage. International Journal of Heritage Studies, 23(5), 408-420.

The Sydney Morning Herald. (2006, 5 July). B. B. King Farewells Montreux. https:// www.smh.com.au/entertainment/music/bb-king-farewells-montreux-20060705gdnwc1.html

Vawda, S. (2019). Museums and the epistemology of injustice: From colonialism to decoloniality. Museum International, 71(1-2), 72-79. https://doi.org/10.1080/13500775. 2019.1638031

Vergo, P. (1989). The new museology. University of Chicago Press.

Vesna, V. (2007). Database aesthetics: Art in the age of information overflow. University of Minneapolis Press.

Wang, X., Jeong, D. H., Dou, W., Lee, S.-W., Ribarsky, W., \& Chang, R. (2009). Defining and applying knowledge conversion processes to a visual analytics system. Computers \& Graphics, 33(5), 616-623.

Whitelaw, M. (2015). Generous interfaces for digital cultural collections. Digital Humanities Quarterly, 9(1), 1-15. 


\section{Sarah Kenderdine}

Windhager, F., Federico, P., Mayr, E., Schreder, G., \& Smuc, M. (2016). A review of information visualization approaches and interfaces to digital cultural heritage collections. In W. Aigner, G. Schmiedl, K. Blumenstein, \& M. Zeppelzauer (Eds.), Proceedings of the 9th Forum Media Technology 2016, St. Pölten, Austria. http://ceur-ws.org

Windhager, F., Federico, P., Schreder, G., Glinka, K., Dörk, M., Miksch, S., \& Mayr, E. (2019). Visualization of cultural heritage collection data: State of the art and future challenges. IEEE Transactions on Visualization and Computer Graphics, 25(6), 2311-2330. 